\title{
ON STAR POLYNOMIALS, GRAPHICAL PARTITIONS AND RECONSTRUCTION
}

\author{
E.J. FARRELL and C.M. DE MATAS \\ Department of Mathematics \\ University of West Indies \\ St. Augustine, Trinidad \\ (Received April 29, 1986)
}

ABSTRACT. It is shown that the partition of a graph can be determined from its star polynomial and an algorithm is given for doing so. It is subsequently shown (as it is well known) that the partition of a graph is reconstructible from the set of nodedeleted subgraphs.

KEY WORDS AND PHRASES. star, star polynomial, star cover, graphical partition, reconstruction.

AMS SUBJECT CLASSIFICATION CODES. 05A99, $05 C 99$.

1. INTRODUCTION.

The graphs here will be finite, undirected, and will have no loops or multiple edges. We define an m-star $\mathrm{S}_{\mathrm{m}}$ to be a tree consisting of a node of valency $\mathrm{m}$ (called the centre of $\mathrm{S}_{\mathrm{m}}$ ) joined to $\mathrm{m}$ other nodes. A 0-star is a node and a 1-star is an edge.

Let $G$ be a graph. A star-cover (or simply, a cover) of $G$ is a spanning subgraph whose components are all stars. Let us associate with each m-star in G, an indeterminate or weight $\mathrm{w}_{\mathrm{m}+1}$; and with each star cover $\mathrm{C}$ consisting of $\mathrm{s}_{\mathrm{m}_{1}}, \mathrm{~S}_{\mathrm{m}_{2}}$, $\ldots, \mathrm{s}_{\mathrm{m}_{\mathrm{k}}} ;$ the weight

$$
w(C)=\prod_{i=1}^{k} w_{m_{i}} .
$$

Then the star polynomial of $G$ (relative to the given weight assignment) is

$$
E(G ; \underline{w})=\Sigma w(C),
$$

where the summation is taken over all the star covers in $G$ and $\underline{w}=\left(w_{1}, w_{2}, \ldots\right)$ is a general weight vector. The basic results on star polynomials are given in the introductory paper by Farre11 [1].

We will show that $\pi_{G}$ - the partition of a graph $G$ can be obtained from $E(G ; \underline{w})$. This will then be used to show that $\Pi_{G}$ is node-reconstructible, a result that can be established by more elementary means (see Tutte [2]). 
For brevity, we will wite $E(G)$ for $E(G ; \underline{w})$, since the same weight vector $\underline{w}$ will be used throughout the paper. Also, in partitions, we will use $r^{k}$ to denote the occurence of $k r^{\prime} s$. Finally, we will assume that $(\underset{r}{n})=0$, for all $r>n$.

2. STAR POLYNOMIALS AND GRAPHICAL PARTITIONS.

First of all, we state a lemma which can be easily proved.

LEMMA 1. Let $v$ be a node of valency $d$ in $G$. Then $G$ contains $\left(\begin{array}{l}d \\ m\end{array}\right)$ m-stars with centre $\mathrm{v}$.

DEFINITION. Let $G$ be a graph with $p$ nodes. A simple m-cover of $G$ is a cover consisting of an $\mathrm{m}-\mathrm{star}$ and $\mathrm{p}-\mathrm{m}-1$ isolated nodes.

It is clear that a simple m-cover in $G$ will have weight $w_{1}^{p-m-1} w_{m+1}$ in $E(G)$. A monomial of this form in $\mathrm{E}(\mathrm{G})$ will be referred to as a simple term, and its coefficient, which will be denoted by $c_{m}$ - a simple coefficient. $c_{m}$ will be the number of simple $m$-covers in $G$. Note that the term $w_{p}$ will also be a simple term.

LEMMA 2. Let $G$ be a graph with $p$ nodes. Let $\Pi_{G}=\left(n^{b_{n}}, \ldots, 2^{b_{2}}, 1^{b_{1}}, 0^{b_{0}}\right)$ $(0 \leq n \leq p-1)$. Then for $m>1$,

$$
c_{m}=\sum_{r=m}^{n}\left(\begin{array}{l}
r \\
m
\end{array}\right) b_{r}
$$

PROOF. This is straightforward.

The following lemma can be easily proved.

LEMMA 3. Let $\mathrm{n}$ be the largest valency of a node in $G$. Then $E(G)$ contains all the terms $w_{1}^{p-r-1} w_{r+1}(0 \leq r \leq n)$ with non-zero coefficient. i.e $c_{r} \neq 0$ for for $(0 \leq \mathrm{r} \leq \mathrm{n})$.

Suppose that we put $n=1$ in the above Lemma. Then $G$ will consist of a set of disjoint edges and possibly isolated nodes. Clearly then the partition of $G$ will be given by

$$
\Pi_{G}=\left(1^{c_{1}}, 0^{p-2 c_{1}}\right)
$$

Hence $\Pi_{G}$ can be found from $E(G)$.

If $\mathrm{n}>1$, then from Lemma 2, we get

$$
\begin{aligned}
c_{k} & =\sum_{r=k}^{n}\left(\begin{array}{l}
r \\
k
\end{array}\right) b_{r}=b_{k}+\sum_{r=k+1}^{n}\left(\begin{array}{l}
r \\
k
\end{array}\right) b_{r}, \quad \text { for } k>1 . \\
\Rightarrow b_{k} & =c_{k}-\sum_{r=k+1}^{n}\left(\begin{array}{l}
r \\
k
\end{array}\right) b_{r} .
\end{aligned}
$$

Therefore $b_{k}$ can be obtained from $c_{k}$ (which is defined in $E(G)$ ) and all the higher b's i.e. $b_{k+1}, b_{k+2}, \ldots, b_{n}$. For $k=1$ and 0 , we can find $b_{1}$ and $b_{0}$ as follows: $c_{1}$ is the number of edges in $G$. Therefore the sum of the valencies of the nodes of $G$ is $2 c_{1}=\sum_{k=1}^{n} k_{k}=b_{1}+\sum_{k=2}^{n} k_{k}$.

$$
\mathrm{b}_{1}=2 \mathrm{c}_{1}-\sum_{\mathrm{k}=2}^{\mathrm{n}} \mathrm{kb}_{\mathrm{k}} \text {. }
$$


$\mathrm{b}_{0}$ is the number of isolated nodes in $\mathrm{G}$. Therefore

$$
b_{0}=p-\sum_{i=1}^{n} b_{i} .
$$

Our discussion yields the following theorem.
THEOREM 1. Let $G$ be a graph with $p$ nodes and let $\pi_{G}=\left({ }^{b}, \ldots, 2^{b}, 1^{b}, 0^{b}\right)$, where $0 \leq n \leq p-1$. Then

$$
\begin{aligned}
& b_{k}=c_{k}-\sum_{r=k+1}^{n}\left(\begin{array}{l}
r \\
k
\end{array}\right) b_{r}, \quad \text { for } \quad 1<k \leq n . \\
& b_{1}=2 c_{1}-\sum_{r=2}^{n} r b_{r}
\end{aligned}
$$

and

$$
\mathrm{b}_{0}=\mathrm{p}-\mathrm{\sum}_{\mathrm{E}=1}^{\mathrm{n}} \mathrm{b}_{\mathrm{r}}
$$

For $n=1, \pi_{G}$ is given by Equation (2.1). For $n=0$, the result is trivial.

Theorem 1 yields an algorith for obtaining $\Pi_{G}$ from $E(G)$. This algorithm is illustrated in the following example:

EXAMPLE 1. Let $G$ be a graph such that

$$
E(G)=w_{1}^{6}+6 w_{1}^{4} w_{2}+7 w_{1}^{3} w_{3}+w_{1}^{2} w_{4}+8 w_{1}^{2} w_{2}^{2}+12 w_{1} w_{2} w_{3}+w_{2}^{3}+w_{2} w_{4}+3 w_{3}^{2 \sim}
$$

First of all, we observe from the term $w_{1}^{6}$, that $G$ has 6 nodes i.e. $p=6$. The simple terms in $E(G)$ are $6 w_{1}^{4} w_{2} 7 w_{1}^{3} w_{3}$ and $w_{1}^{2} w_{4}$. Therefore $c_{1}=6, c_{2}=7$ and $c_{3}=1$. Since the largest $k$ for which $w_{k}$ occurs in $E(G)$ is 4 , it follows that $n=3$. Now

$$
\begin{aligned}
& b_{3}=c_{3}=1 ; b_{2}=c_{2}-\sum_{r}^{3}\left(\begin{array}{l}
r_{2} \\
)
\end{array} b_{r}=7-3 b_{3}=7-3=4 .\right. \\
& b_{1}=2(6)-\sum_{k=2}^{3} k b_{k}=12-2 b_{2}-3 b_{3}=12-8-3=1 . \\
& b_{0}=p-\sum_{r=1}^{3} b_{r}=6-1-4-1=0 .
\end{aligned}
$$

Hence $\Pi_{G}=\left(3^{l} 2^{4} 1^{1}\right)$.

It would be nice to be able to obtain $G$ itself from $E(G)$. From Theorem 1 , $\Pi_{G}$ can be obtained. However there can be several graphs with the same partition. Since only the simple terms in $E(G)$ are used to obtain $\Pi_{G}$, it is not surprising that $G$ itself is not clearly defined. Should $G$ itself be clearly defined by the simple terms, then it would mean that the remaining terms of $E(G)$ are useless as far as the characterization of $\mathrm{G}$ is concerned. It would be interesting to investigate the nature of these 'useless terms'.

Suppose that $\Pi_{G}$ is unigraphic (i.e. there is only one graph with partition $\Pi_{G}$ ), then $G$ could be uniquely constructed from $\pi_{G^{*}}$. Hence we have the following theorem. THEOREM 2. Let $\Pi_{G}$ be unigraphic. Then $G$ can be constructed from $E(G)$. PROOF. This follows from Theorem 1 and the discussion above. 
THEOREM 3. Let $G$ and $H$ be two graphs with $p$ nodes. Then $\Pi_{G}=\Pi_{H}$ if and only if $E(G)$ and $E(H)$ have the same simple coefficients.

PROOF. Suppose that the simple coefficients in $E(G)$ and $E(H)$ are equal. Then from Theorem 1, G and $H$ must have the same partition. Conversely, suppose that $\Pi_{G}=\Pi_{H}$. Then from Lemma 2, the coefficients $c_{r}(r>1)$ in $E(G)$ and $E(H)$ must be equal. Finally, $c_{0}=1$, for all graphs. Hence $E(G)$ and $E(H)$ have the same simple coefficients. The result therefore follows.

3. STAR POLYNOMIALS AND RECONSTRUCTION.

The following theorem is analogous to the result for circuit polynomials given in Lemma 3 of Farrell and Gre11 [3], with $i=1$. We suspect that the general result holds for all F-polynomials (see Farre11 [4]). Here G-x denotes the graph obtained from $G$ by removing node $x . V(G)$ is the node set of $G$. THEOREM 4.

$$
\frac{\partial E(G)}{\partial w_{1}}=\sum_{x \in V(G)} E(G-x ; \underline{w})
$$

PROOF. Let us write $E(G)=\sum_{j} A_{j} w_{1}{ }^{n}, j w_{2}{ }^{n_{2}}, j \ldots w_{p}{ }^{n}, j$, where $p$ is the number of nodes in $G$. Then

$$
\frac{\partial E(G)}{\partial w_{1}}=\sum_{j} n_{1, j} A_{j} w_{1}^{n_{1}, j=1}{ }_{w_{2}}^{n_{2}, j} \ldots w_{p}^{n}{ }^{n}, j
$$

It is clear that the monomial $w_{1}{ }^{n}, j-\underset{w_{2}}{n_{2}} \ldots w_{p}^{n}, j$ is the weight of a cover with one isolated node less than the corresponding cover in $G$. It is therefore the weight of a cover in G-x, for some node $x$ in $G$. Hence it is a monomial of the polynomial $\sum \mathrm{E}(\mathrm{G}-\mathrm{x} ; \underline{w})$. Conversely, every cover of $\mathrm{G}-\mathrm{x}$ can be extended to a cover of $\mathrm{G}$ by adding an isolated node. Therefore every monomial $\mathrm{m}$ in $\sum \mathrm{E}(\mathrm{G}-\mathrm{x} ; \underline{w})$ yields a corresponding monomial $w_{1} m$ in $G$. The derivative of $w_{1} m$ with respect to $w_{1} y i e l d s$ a term with monomial m. It follows that $\frac{\partial E(G)}{\partial w_{1}}$ and $\sum E(G-x ; w)$ have the same monomials.

Since $A_{j}$ is the coefficient of ${ }_{w_{1}}^{n_{1}}, j_{w_{2}}^{n_{2}, j \ldots w_{p}}{ }^{n}, j, G$ has $A_{j}$ covers consisting of $n_{1, j}$ isolated nodes $n_{2, j}$ edges,..., $n_{p, j}(p-1)$ - stars. Suppose that node $x$ is removed from G. Then $G-x$ will have a similar cover but with $n_{1, j}-1$ isolated nodes. Since node $x$ could be any of the $n_{1, j}$ isolated nodes in the cover, it follows that each such cover in $G$ gives rise to $n_{1, j}$ covers with one less isolated node. Hence the coefficient of $w_{1}{ }^{n_{1}, j}{ }^{-1} w_{2}{ }^{2}, j \ldots w_{p} p, j$ in $\sum E(G-x ; \underline{w})$ is $n_{1, j} A_{j}$. From Equation (3.1) it follows that the monomials occur in $\sum E(G-x ; \underline{w})$ and $\frac{\partial E(G)}{\partial w_{1}}$ with equal coefficients. Hence the result follows.

Throughout the rest of this section, we will assume that the graph has at least three nodes. Also 'reconstructible' would mean node-reconstructible. By the deck 
$D_{G}$ a graph $G$ we would mean the set $\{G-x: \times \varepsilon V(G)\}$.

It is well known (see Harary [5], Kel1y [6], and Chatrand and Kronk [7]) that disconnected graphs are reconstructible. It follows that $\Pi_{G}$ is reconstructible if $G$ is disconnected, and therefore the number of isolated nodes in $G$ is reconstructible. We can however, prove the latter independently. The following lemma gives a connection between the number of isolated nodes and $\mathrm{D}_{\mathrm{G}}$.

LEMMA 4. Let $G$ be a graph with $p$ nodes. Then $G$ has $r(>0)$ isolated nodes if and only if $D_{G}$ has exactly $r$ graphs with $r-1$ isolated nodes and $p-r$ graphs with $\mathrm{r}$ or more isolated nodes.

PROOF. Suppose that $G$ has $r$ isolated nodes. Then $D_{G}$ must be of the form described. Conversely, suppose that $D_{G}$ is as described in the theorem. Let $k$ be the number of isolated nodes in $G$. Since every element of $D_{G}$ has at least $r-1$ isolated nodes, $G$ cannot have less than $r-1$ isolated nodes or it would mean that the removal of each node from $G$ yields at least one new isolated node, and this is impossible unless $G$ is a matching (in which case the result holds). Therefore $k \geq r-1$. Since $D_{G}$ contains no elements with less that $r-1$ isolated nodes, $k \neq r-1$. Hence $k>r-1$. But exactly $r$ elements of $D_{G}$ has $r-1$ isolated nodes and only one node can be removed at a time from $G$ to form an element of $D_{G}$. Therefore $G$ has exactly $r$ nodes each of whose removal reduces $k$ to $r-1$. These nodes must be themselves isolated nodes. $\Rightarrow k \geq r$. Clearly $k \ngtr r$. Therefore $k=r$ and the result follows.

From the above lemma, we see that $b_{0}$ (of Theorem l) can be obtained from $D_{G}$. It follows that if $D_{G}$ is given, then we can find $\pi_{G}$ - provided that all the remaining $\mathrm{b}_{\mathrm{r}}$ 's $(0<\mathrm{r} \leq \mathrm{n})$ can be determined. The following result is well known (see Tutte [2]). We will give different derivation using star polynomials. THEOREM 5. $\Pi_{G}$ is reconstructible.

PROOF. Let $G$ be a graph with $p$ nodes. From Theorem 4, we have, by integrating both sides with respect to $w_{1}$,

$$
E(G)=\int(\Sigma E(G-x ; \underline{w}))+C\left(w_{2}, w_{3}, \ldots, w_{p}\right),
$$

where $\mathrm{C}\left(\mathrm{w}_{2}, \mathrm{w}_{3}, \ldots, \mathrm{w}_{\mathrm{p}}\right)$ is a polynomial in the weights $\mathrm{w}_{2}, \mathrm{w}_{3}, \ldots, \mathrm{w}_{\mathrm{p}}$.

Suppose that $D_{G}$ is given. Then $\sum E(G-x ; \underline{w})$ can be found. Hence $\int \sum E(G-x ; \underline{w})$ can be found. But this polynomial contains all the simple terms except ${ }_{p} \cdot{ }^{-}$Therefore all the simple coefficients of $E(G)$, except $c_{p-1}$ can be immediately found. We will consider two cases (i) $c_{p-2}=0$ and (ii) $c_{p-2} \neq 0$.

If $c_{p-2}=0$, then from Lemma $3, c_{p-1}=0$. Therefore all the simple coefficients will be defined. It follows from Theorem 1 , that $\Pi_{G}$ can be found. If $c_{p-2} \neq 0$ then $c_{p-1}$ will be unknown. However $b_{0}$ will be known from $D_{G}$ (Lemma 4). 
Therefore the system of equations in Theorem 1 will have $p-1$ equations and p-1 unknowns. It can be solved to find $b_{1}, b_{2}, \ldots, b_{p-1}$. Hence $\pi_{G}$ can be found.

In the proof of Theorem 5, we did not give any useful practical method for finding $\Pi_{G}$, when $\mathrm{c}_{\mathrm{p}-2} \neq 0$. We shall do so now.

The equations of Theorem 1 can be written as follows:

$$
\begin{aligned}
& p=b_{p-1}+b_{p-2}+\ldots+b_{2}+b_{1}+b_{0} \\
& 2 c_{1}=\left(\begin{array}{c}
p-1 \\
1
\end{array}\right) b_{p-1}+\left(\begin{array}{c}
p-2 \\
1
\end{array}\right) b_{p-2}+\ldots+2 b_{2}+b_{1} \\
& c_{2}=\left(\begin{array}{c}
p-1 \\
2
\end{array}\right) b_{p-1}+\left(\begin{array}{c}
p-2 \\
2
\end{array}\right) b_{p-2}+\ldots+b_{2} \\
& \cdot \\
& c_{p-2}=\left(\begin{array}{c}
p-1 \\
p-2
\end{array}\right) b_{p-1}+\left(\begin{array}{l}
p-2 \\
p-2
\end{array}\right) b_{p-2} .
\end{aligned}
$$

By adding and subtracting alternate equations, we get

$$
\mathrm{p}-2 \mathrm{c}_{1}+\mathrm{c}_{2}-\mathrm{c}_{3} \ldots+(-1)^{\mathrm{p}_{\mathrm{p}-2}}=\mathrm{b}_{0}+(-1)^{\mathrm{p}_{\mathrm{b}-1}} \text {. }
$$

Let us denote the L.H.S. of this equation by S. Then

$$
\begin{aligned}
& \mathrm{S}=\mathrm{b}_{0}+(-1)^{\mathrm{P}_{\mathrm{p}-1}} . \\
\Rightarrow \quad & \mathrm{b}_{\mathrm{p}-1}=(-1)^{\mathrm{p}}\left(\mathrm{S}-\mathrm{b}_{0}\right)
\end{aligned}
$$

Since $S$ can be found from the simple coefficients $c_{r}(r \geq p-2)$ and $p$, and $b_{0}$ can be found (from Lemma 5), $b_{p-2}$ can be found from Equation (3.3). Hence all the b's can be found by using the algorithm suggested by Theorem 1 .

The following example illustrates the technique.

EXAMPLE 2. Let $G$ be a graph with $D_{G}$ given below in Figure 1.

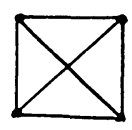

$\mathrm{C}_{1}$

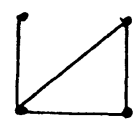

$G_{2}$

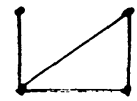

$G_{3}$

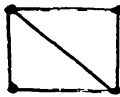

$G_{4}$

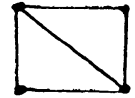

$\mathrm{G}_{5}$

Figure 1.

It can be easily confirmed that

$$
\begin{aligned}
& E\left(G_{1}\right)=w_{1}^{4}+6 w_{1}^{2} w_{2}^{2}+12 w_{1} w_{3}+3 w_{2}^{2}+4 w_{4} \\
& E\left(G_{2}\right)=E\left(G_{3}\right)=w_{1}^{4}+4 w_{1}^{2} w_{2}+5 w_{1} w_{3}+w_{2}^{2}+w_{4} \\
& E\left(G_{4}\right)=E\left(G_{5}\right)=w_{1}^{4}+5 w_{1}^{2} w_{2}+8 w_{1} w_{3}+2 w_{2}^{2}+2 w_{4} .
\end{aligned}
$$

Therefore

$$
\sum_{i=1}^{5} E\left(G_{i}\right)=\frac{\partial E(G)}{\partial w_{1}}=5 w_{1}^{4}+24 w_{1}^{2} w_{2}+38 w_{1} w_{3}+9 w_{2}^{2}+10 w_{4} \text {. }
$$

By integrating with respect to $w_{1}$, we get

$$
E(G)=w_{1}^{5}+8 w_{1}^{3} w_{2}+19 w_{1}^{2} w_{3}+10 w_{1} w_{4}+C\left(w_{2}, w_{3}, w_{4}, w_{5}\right) .
$$


Therefore

$$
\begin{aligned}
\mathrm{p} & =5, \mathrm{c}_{1}=8, \mathrm{c}_{2}=19 \text { and } \mathrm{c}_{3}=10 . \\
\Rightarrow \quad \mathrm{S} & =5-16+19-10=-2 .
\end{aligned}
$$

Since no element of $D_{G}$ has an isolated node, it follows from Lemma 5 that $b_{0}=0$. From Equation (3.3), we get

$$
\begin{aligned}
& \mathrm{b}_{4}=(-1)^{5}(-2-0)=2 . \\
& \mathrm{b}_{3}=c_{3}-\sum_{\mathrm{r}-4}^{4}\left(\begin{array}{l}
\mathrm{r} \\
3
\end{array}\right) \mathrm{b}_{\mathrm{r}}=c_{3}-4 \mathrm{~b}_{4}=10-8=2 . \\
& \mathrm{b}_{2}=\mathrm{c}_{2}-\sum_{\mathrm{r}=3}^{4}\left(\begin{array}{l}
\mathrm{r} \\
2
\end{array}\right) \mathrm{b}_{\mathrm{r}}=\mathrm{c}_{2}-\left(\begin{array}{l}
3 \\
2
\end{array}\right) \mathrm{b}_{3}-\left(\begin{array}{l}
4 \\
2
\end{array}\right) \mathrm{b}_{4}=19-6-12=1 . \\
& \mathrm{b}_{1}=2 \mathrm{c}_{1}-\sum_{\mathrm{r}=2}^{4}\left(\begin{array}{l}
\mathrm{r}_{1} \\
)
\end{array} \mathrm{b}_{\mathrm{r}}=2 \mathrm{c}_{1}-2 \mathrm{~b}_{2}-3 \mathrm{~b}_{3}-4 \mathrm{~b}_{4}=16-2-6-8=0 .\right.
\end{aligned}
$$

Hence we get

$$
\Pi_{G}=\left(4^{3}, 3^{2}, 2^{1}\right) \text {. }
$$

Suppose that $G$ is unigraphic. Then $G$ is uniquely determined from $\Pi_{G^{*}}$ But $\Pi_{G}$ is reconstructible from $D_{G}$ (Theorem 5). It follows that $G$ is uniquely reconstructible from $D_{G}$. Thus we have the following theorem, which can otherwise be proved by more elementary methods.

THEOREM 6. The Reconstruction Conjecture holds for all graphs with unigraphic partitions.

\section{REFERENCES}

1. FARRELL, E. J., On a Class of Polynomials Associated with the Stars of a Graph and its Application to Node Disjoint Decompositions of Complete Graphs and Complete Bipartite Graphs into Stars, Canad. Math. Bu11., 22(1)(1978) 35-46.

2. TUTTE, W. T., "Graph Theory", Encyclopedia of Mathematics and Its Applications, Vo1. 21 Addison-Wesley, 1984.

3. FARRELL, E. J., and GRELL, J. C., On Reconstructing the Circuit Polynomial of a Graph, Caribb. J. Math. 1(3) (1983) 109-119.

4. FARRELl, E. J., On a General Class of Graph Polynomials, J. Comb. Theory B. 26 (1979) 111-122.

5. HARARY, F., On the Reconstruction of a Graph from a Collection of Subgraphs, "Theory of Graphs and its Applications"(M. Fiedler ed.) Gordon and Breach, New York, 1979, 131-146.

6. KELlY, P. J., On Some Mapping Related to Graphs, Pacific J. Math. 24(1964) 191-194

7. CHARTRAND, G. and KRONK, H. V., On Reconstructing Disconnected Graphs, Ann. N. Y. Acad. Sci. $175(1970), 85-86$. 


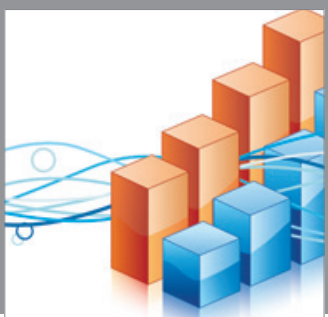

Advances in

Operations Research

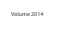

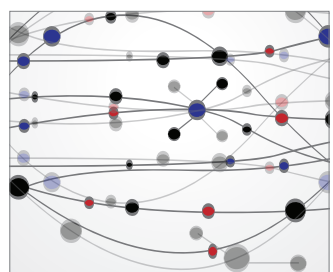

\section{The Scientific} World Journal
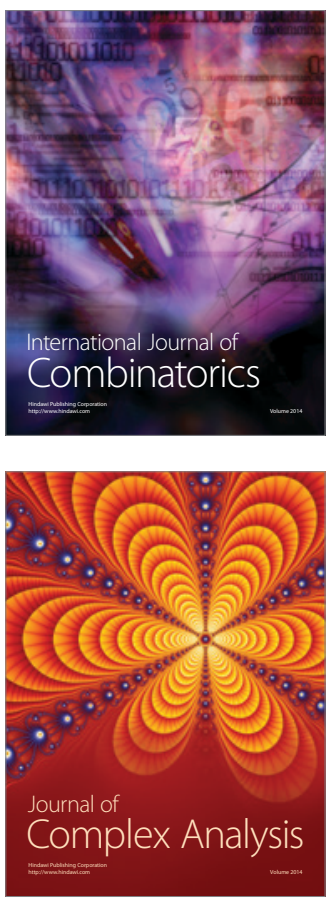

International Journal of

Mathematics and

Mathematical

Sciences
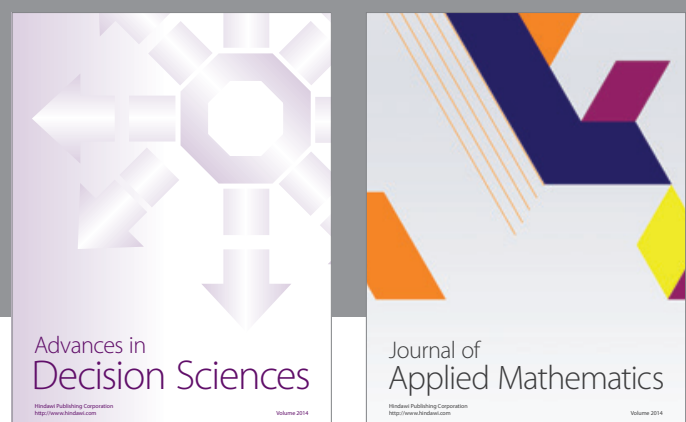

Journal of

Applied Mathematics
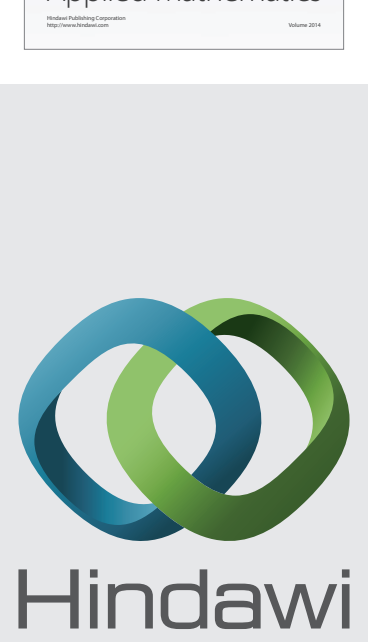

Submit your manuscripts at http://www.hindawi.com
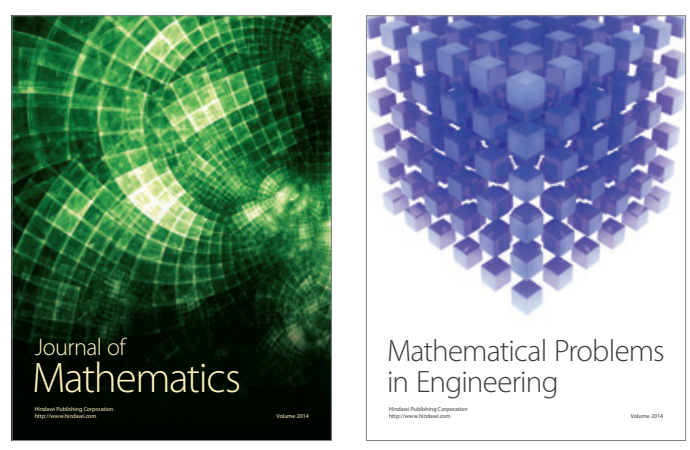

Mathematical Problems in Engineering
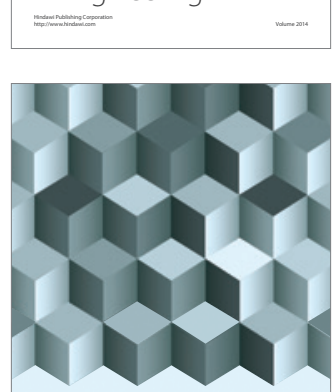

Journal of

Function Spaces
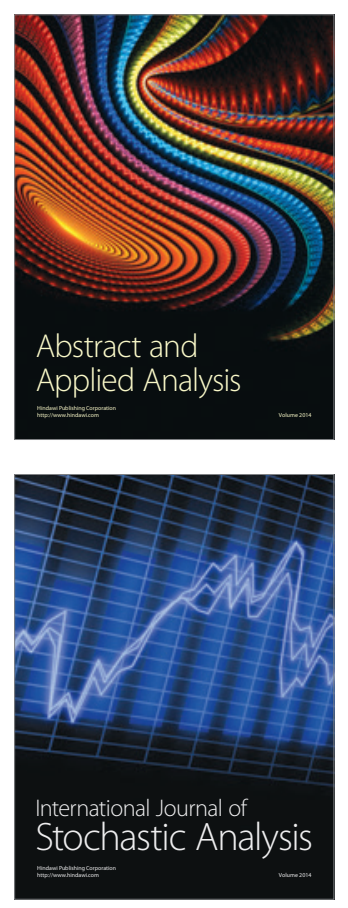

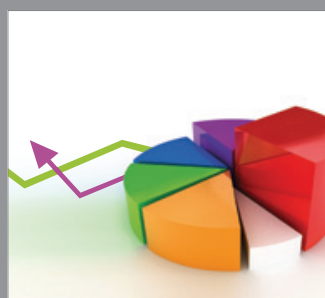

ournal of

Probability and Statistics

Promensencen
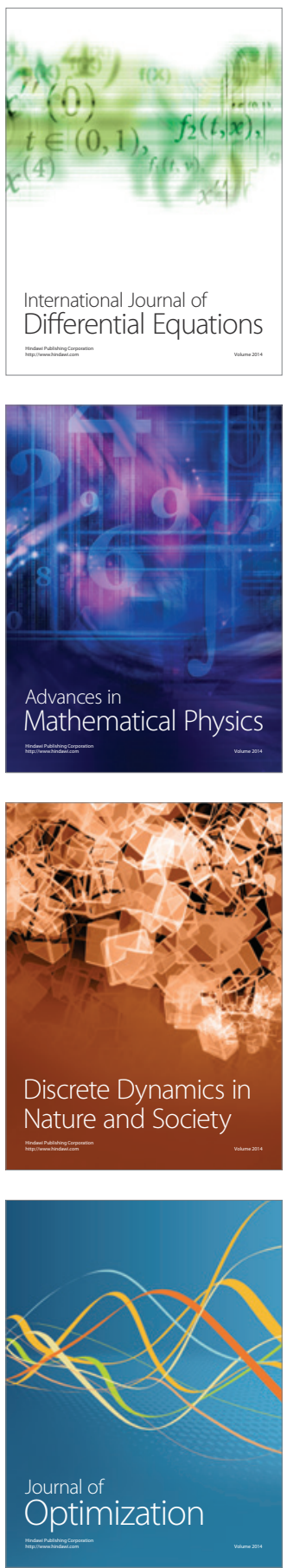